\title{
A study of disease pattern in patients presenting in the emergency department of a tertiary hospital catering to industrial workers.
}

\author{
${ }^{1}$ Jitesh Dhingra, ${ }^{2}$ Girish Arora, ${ }^{3}$ Paschal D' Souza \\ ${ }^{1}$ Junior Resident, Casualty, Accidental and Trauma Services, ESIC Hospital, Basaidarapur. \\ ${ }^{2}$ Casualty Incharge, Accidental and Trauma Services, ESIC Hospital, Basaidarapur. \\ ${ }^{3}$ Professor and Head Dermatology, ESIC Hospital, Basaidarapur.
}

\begin{abstract}
Emergency department (in a health care facility) is a section of an institution that is staffed and equipped to provide rapid and varied emergency care, especially for those who are stricken with sudden and acute illness or who are the victims of severe trauma.

This study analyzed the disease pattern of patients presenting to the emergency department of a tertiary care insurance based hospital in Delhi. The results showed maximum patients attended were under the Medicine and Surgery discipline. A large proportion of these patients were non emergency cases that could have been managed in the Outpatient Department (OPD).

As the cause of overcrowding of the hospital emergency department was attributed to the number of people presenting to the emergency department with non emergent problems it discusses the steps that can be taken to better channelize the use of emergency medical services.
\end{abstract}

\section{Introduction}

Emergency Care should be of high quality, cost effective and compassionate. The Accident \& Emergency services are one of the mainstays in the chain of medical care offered by the present day hospitals. The need for effective emergency healthcare delivery through an Emergency Department (ED) is well recognized and all hospitals must be able to provide basic life support through their Emergency Services to the patients in need.

Major building blocks of any successful Emergency Care are:

1. Availability of adequate resources of all life saving drugs and surgical items.

2. Immediate professional attention after arrival.

3. Continued and constant medical support till the patient is in the ED.

4. Speedy diagnosis and resuscitation.

The ED of modern hospitals are considered as "mini-hospitals" today and form an important part in the chain of medical care. The ED of ESI hospital caters to about 300 patients, admits about 20-25 patients daily.

Since emergency care requires intensive input of material and manpower it should be optimally utilized for the management of patients requiring urgent medical care which cannot be met by the regular outpatient departments due to inappropriateness of time or lack of emergency care facilities. Improper utilization of these facilities may cause great strain on these services causing lack of effective care to deserving patients. In this context this study was undertaken to analyze the pattern of patients availing Emergency facilities at a tertiary care ‘Employee State Insurance Scheme Corporation' (ESIC) hospital in Delhi.

The key objectives were to:

\section{Aims And Objectives:}

1. Determine the workload of the hospital Emergency Department (Casualty) and find out trends over a specified period of time (4 months) in terms of number of patients as well as available emergency beds.

2. Ascertain the category of patients (attending Casualty) based on the type of illness they are diagnosed to have during the specified period of time. 


\section{Material And Methods:}

A retrospective record based study was carried out of the patients who had reported to the Emergency Department (casualty) of the biggest ESI hospital of India, ESIC, Basaidarapur over a period of 4 months (October 2012-January 2013).

Study design was cross sectional and study population included all patients attending Emergency Department during the four months study period. Data was collected and patients categorized on the basis of the specialty services they required at the time of presentation such as:

1. Medicine

2. Surgery

3. Pediatrics

4. ENT

5. Ophthalmology

6. Orthopedics

Data about admissions and patient load in different shifts was also collected.

\section{Results}

It is seen that on an average about 20-25 patients are admitted daily from the Casualty. During the study, it was found out that most of the admissions took place in the afternoon shift ( $2 \mathrm{pm}$ to $8 \mathrm{pm}$ ) and least admissions were made in the night shift. (Figure 4)

Of the total number of admissions daily on an average 11-14 patients were admitted under the discipline of medicine.

The number of patients admitted in Orthopedics and Surgery were on an average 6-8.

It was also found that there was significant increase in the number of admissions in the month of October (1151) which was the month of dengue outbreak in Delhi. (Figure 1)

The total number of patients managed in the Emergency Department over a period of 4 months and their respective percentage distribution under various departments was as follows: (Figure 2)

Medicine: 12128 (56.08\%)

Surgery: $3166(14.64 \%)$

Orthopedics: $2519(11.64 \%)$

Pediatrics: $2945(13.61 \%)$

ENT: $525(2.42 \%)$

Eye: $302(1 \%)$

Dental: $39(0.18 \%)$

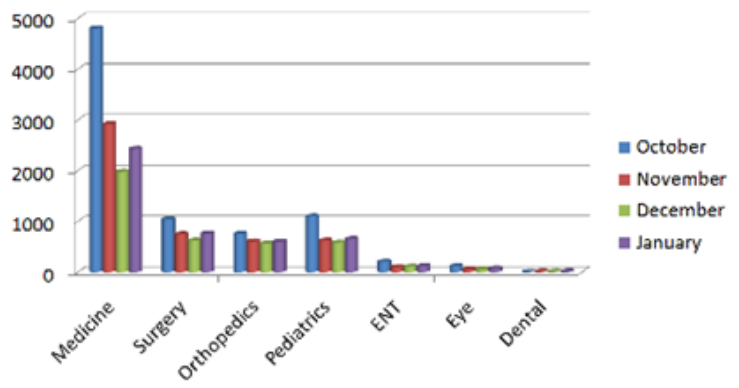

Figure 1

Figure 1: The bar diagram shows a graphical representation of the number of patients attending casualty of ESIC hospital, Basaidarapur under various disciplines over a period of $\mathbf{4}$ months. 
Table 1

\begin{tabular}{|l|l|l|l|l|}
\hline & October & November & December & January \\
\hline Medicine & 4805 & 2918 & 1978 & 2427 \\
\hline Surgery & 1039 & 746 & 619 & 762 \\
\hline Orthopedics & 752 & 605 & 560 & 602 \\
\hline Pediatrics & 1096 & 619 & 580 & 650 \\
\hline ENT & 202 & 99 & 106 & 118 \\
\hline Eye & 115 & 57 & 58 & 72 \\
\hline Dental & 4 & 12 & 9 & 14 \\
\hline
\end{tabular}

Table 1: The table shows the total number of patients attending casualty of ESIC hospital, Basaidarapur over a period of 4 months under various disciplines.

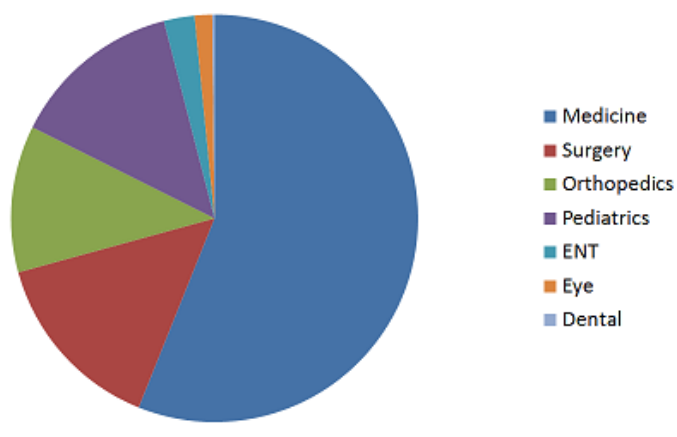

Figure 2

Figure 2: A pie chart representation of the percentage wise distribution of the total number of patients attending casualty.

Table 2

\begin{tabular}{|l|l|l|}
\hline & Total Number of Cases & Percentage distribution (\%) \\
\hline Medicine & 12128 & 56.08 \\
\hline Surgery & 3166 & 14.64 \\
\hline Orthopedics & 2519 & 11.64 \\
\hline Pediatrics & 2945 & 13.61 \\
\hline ENT & 525 & 2.42 \\
\hline Eye & 302 & 1.00 \\
\hline Dental & 39 & 0.18 \\
\hline
\end{tabular}

Table 2: The table shows the total number of patients attended under various disciplines over a period of 4 months in ESIC hospital casualty.

The bulk of the patients managed in the Emergency Department are kept under observation under the discipline of Medicine (56.08\%). About $45 \%$ of these patients are found to be suffering from respiratory disorders, the top ailment being Chronic Obstructive Airways Disease (COAD). The other frequent cause of admission under Medicine is Chronic Kidney Disease.

It was found significant number of patients were suffering from chest pain(both cardiac and respiratory),headache and loose motions.

Besides medical emergencies a significant number was of surgical and orthopedic emergencies with a large proportion of these emergencies being road traffic accidents $(22.05 \%)$ and fall from height $(34.01 \%)$. The other surgical emergencies were renal colic, cholecystitis and Koch's abdomen presenting with vague complaints of pain abdomen.

A large proportion of medico-legal cases were crush injuries and factory injuries (19.43\%). 


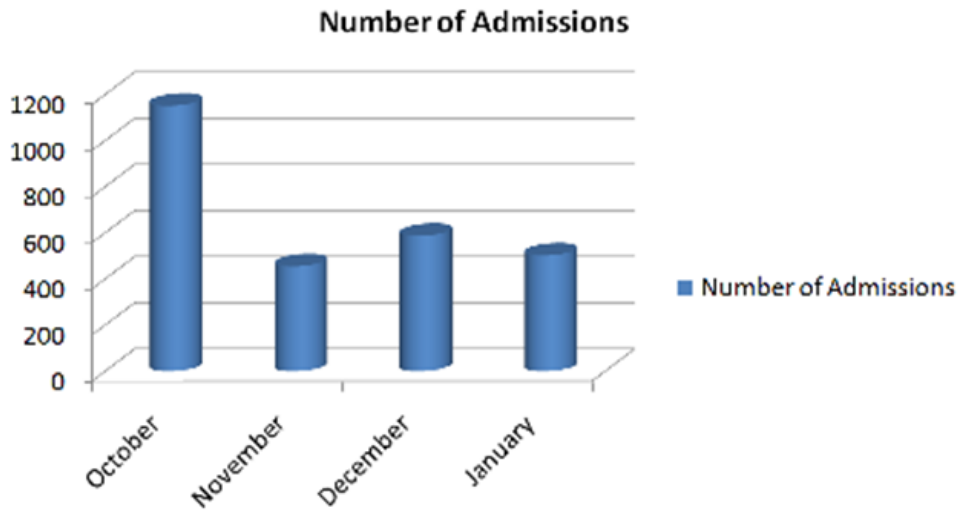

Figure 3

Figure 3: A graphical representation of the total number of admissions in the casualty department over a period of 4 months.(October 2012- January 2013)

Table 3

\begin{tabular}{|l|l|}
\hline & Number of Admissions \\
\hline October & 1151 \\
\hline November & 455 \\
\hline December & 587 \\
\hline January & 502 \\
\hline
\end{tabular}

Table 3: The table shows the total number of admissions in ESIC Hospital Casualty over a period of 4 months.

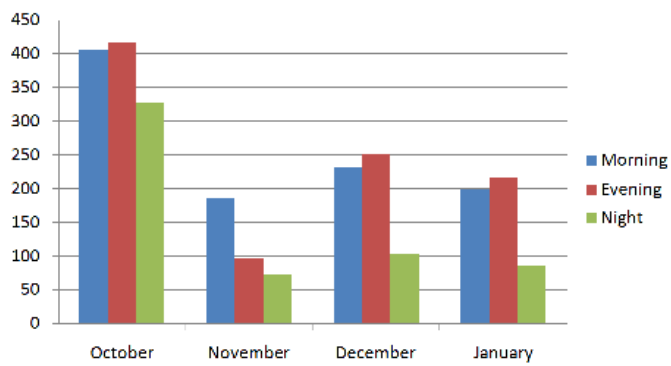

Figure 4

Figure 4: This graphical representation shows the total number of admissions over a period of 4 months with further distribution of admissions split into morning (8 a.m. to 2 p.m.), evening (2 p.m. to 8 p.m.) and night shift (8 p.m. to 8 a.m.) admissions.

Table 4

\begin{tabular}{|l|l|l|l|}
\hline & Morning & Evening & Night \\
\hline October & 406 & 417 & 328 \\
\hline November & 185 & 97 & 73 \\
\hline December & 231 & 252 & 104 \\
\hline January & 200 & 216 & 86 \\
\hline
\end{tabular}

Table 4: The table shows the shift wise (morning, evening and night shift) distribution of the number of admissions in the emergency department over a period of 4 months. 


\section{Discussion:}

The percentage wise distribution of patients managed in the Emergency Department of ESIC Hospital were categorized under assigned disciplines were: Medicine- $56.08 \%$, Surgery-14.64\% ,Othopedics$11.64 \%$,Pediatrics-13.61\%,ENT-2.42\%,Eye-1\%. In comparison to a similar study done in Safdarjung Hospital in the year 1990 the specialty-wise distribution of the patients in descending order was Medicine, $(29.27 \%)$, Surgery (26.53\%),Orthopedics (16.47\%) and Pediatric Medicine (11.33\%). In their study a category 'Others' included patients who have E.N.T., eye or dental problems. ${ }^{1}$

Analyzing the results obtained in our hospital some conclusions can be drawn as mentioned below:

1. The possible explanation for the difference in admission rates could be that during morning shift even though the OPD is open, most patients prefer to not go there to save time and decrease waiting time and so in the morning many patients who could have attended outpatient departments attend casualty as casualty attainders. The waiting time is increasing because of an increase in the number of insured persons which is not only because of the increase in population but also because the salary limit of people covered in ESI has also risen. The limited beds available get filled up during this shift, leaving very few beds for patients attending ED during night shift.

2. It was also found that if the casualty is not to be reduced to doing the work of the regular outpatient department, some disposition of the casual attainder has to be made. The reasons for casual use of the emergency department may be many, some of them being - non availability of dispensary services, for example, on holidays; the patients feels that the hospital has better facilities for the treatment ${ }^{2}$. The patient may find the hospital more convenient to visit or just wants a second opinion ${ }^{3}$.

3. Casual visitors will continue to arrive in the casualty and emergency department. There exists a wide disagreement as to how these patients were to be managed. One view holds that the doors of the casualty department should be closed to these patients in order to increase the efficiency in dealing with the real emergencies ${ }^{4}$.

A more liberal stand has been taken by another researcher who states that no patient should be refused from treatment, however trivial his complaint may seem. Such a patient may be referred to his department, if he has one, or be treated in the department and subsequently be followed up in OPDs. ${ }^{5}$

4. Majority of the patients were admitted under the discipline of General Medicine. Of these, chronic kidney disease and respiratory disorders accounted for the maximum number of admissions. Another category of patients that formed a major number were chest pain cases both of cardiac and gastric origin. Co morbidities in the form of Diabetes Mellitus and Hypertension are common.

5. The study also shows us the seasonal variations in the number of patients attending casualty. There was as significant increase in the number of patients attending casualty in the month of October which was the month of dengue outbreak in Delhi. There is also a huge increase in the number of admissions in the dengue season with the number of admissions being almost double. Number of patients attending under the specialty of Medicine rose by $58 \%$ in the dengue season.

6. The study reveals that a majority of patients attending casualty of ESIC are cases of respiratory problems, the reason for this probably being that most of the beneficiaries are working in an industrial environment and are exposed to allergens, dust and chemicals which they are constantly inhaling.

\section{References}

[1]. S. Malhotra and R.S. Gupta A study of the workload of the casualty department of a large city hospital (Safdarjung Hospital) Health and Population - Perspectives \& Issues 15(1\&2):68-76, 1992.

[2]. PatelAR (1971) Modes of Admission to Hospital, A Survey of Emergency Admissions to a General Medical Unit.British Medical Journal,1: p;281 -283.

[3]. Griffiths W, King P, Preston B. Casualty Department or GP. Service? British Medical Journal 1967;3: 46

[4]. Black well B. Why patients come to a casualty department. The Lancet 1962;1: 369-71.

[5]. Grant, Cohen The Emergency Department. Hospital Management. Churchill Livingstone, Edinburgh, London and New York; 1973,p. 115. 